Arthroskopie $2020 \cdot 33: 412-419$

https://doi.org/10.1007/s00142-020-00385-z

Online publiziert: 31 . Mai 2020

(c) Der/die Autor(en) 2020

\section{P. Kappel' • M. Liebensteiner ${ }^{2}$ AGA Komitee Knie-Patellofemoral}

'Klinik für Orthopädie, Unfallchirurgie und Sporttraumatologie, Krankenhaus Köln-Merheim, Köln, Deutschland

${ }^{2}$ Universitätsklinik für Orthopädie Innsbruck, Medizinische Universität Innsbruck, Innsbruck, Österreich

\title{
Bildgebende Diagnostik patellofemoraler Störungen
}

Patellofemorale Störungen wie die Instabilität der Kniescheibe oder Patellofemoralarthrose sind häufige Krankheitsbilder, die mit einer erheblichen Morbidität einhergehen. Zur diagnostischen Abklärung patellofemoraler Störungen gehört neben einer spezifischen Anamnese und detaillierten, körperlichen Untersuchung auch die bildgebende Diagnostik. Hier kommen, abhängig von Anamnese und klinischem Befund, verschiedene Bildgebungsverfahren zur Anwendung. Das Ziel dieses Artikels ist es, die verschiedenen bildgebenden Techniken darzustellen, die zur Diagnostik patellofemoraler Störungen angewandt werden und die darin ersichtlichen pathologischen Befunde zu diskutieren.

\section{Röntgendiagnostik}

Die Basisdiagnostik patellofemoraler Störungen stellt das Röntgen in 3 Ebenen dar. Neben den Standardebenen anterior-posterior und lateral wird auch eine Tangentialaufnahme der Patella durchgeführt. Auf der einen Seite können hier knöcherne Traumafolgen und degenerative Veränderungen des $\mathrm{Pa}$ tellofemoralgelenks (PFG) dargestellt werden, auf der anderen Seite kann die Stellung der beiden Gelenkpartner zueinander beurteilt werden.

\section{Kniegelenk anterior-posterior}

Die a.-p.-Aufnahme des Kniegelenks dient v. a. der Darstellung des Femorotibialgelenks. Sowohl Frakturen als auch degenerative Veränderungen können gezeigt werden. Um degenerative Veränderungen bestmöglich abzubilden, sollte die Aufnahme unter Belastung durchgeführt werden (Rosenberg-Aufnahme). Darüber hinaus kann auch die Stellung der Patella in mediolateraler und kraniokaudaler Ausrichtung beurteilt werden. Eine verlässliche Aussage zur Patellaposition ist jedoch nur bei streng anterior-posteriorer Einstellung des Gelenks möglich.

\section{Kniegelenk lateral}

In dieser Aufnahme kann der Gelenkspalt des PFG eingesehen werden und somit Frakturen, z. B. der Patella, und degenerative Veränderungen gut beurteilt werden. Durch einen entsprechenden Weichteilschatten kann ein großer Gelenkerguss auch im Röntgen zur Darstellung kommen. Darüber hinaus können in dieser Projektion verschiedene, für eine patellofemorale Instabilität prädisponierende Faktoren evaluiert werden. Um entsprechende Messungen durchführen zu können, ist allerdings eine streng seitliche Aufnahme obligat (Kongruenz der posterioren Kondylen), da bereits eine Rotation von $5^{\circ} \mathrm{zu}$ Missinterpretationen führen kann [12].

\section{) Die Insall-Savati-Ratio wird im klinischen Alltag international am häufigsten verwendet}

Zunächst kann die Höhe der Patella bestimmt werden, um eine Patella alta zu identifizieren. Die Definition der Patella alta erfolgt hierbei uneinheitlich. Bie- dert et al. fanden in der Literatur 18 verschiedene Messmethoden [3]. Die vier in der Literatur am häufigsten angewandten Messmethoden sind die Insall-SavatiRatio (ISR), der Caton-Deschamps-Index (CDI), die modifizierte Insall-Salvati-Ratio (mISR) und der BlackburnePeel-Index (BPI), wobei die ISR im klinischen Alltag international am häufigsten verwendet wird [21]. Bei der ISR wird das Verhältnis der Patellalänge (größte diagonale Länge) zur Länge des Ligamentum patellae berechnet, beim CDI das Verhältnis der Länge der Patellagelenkfläche zur Strecke zwischen dem un-

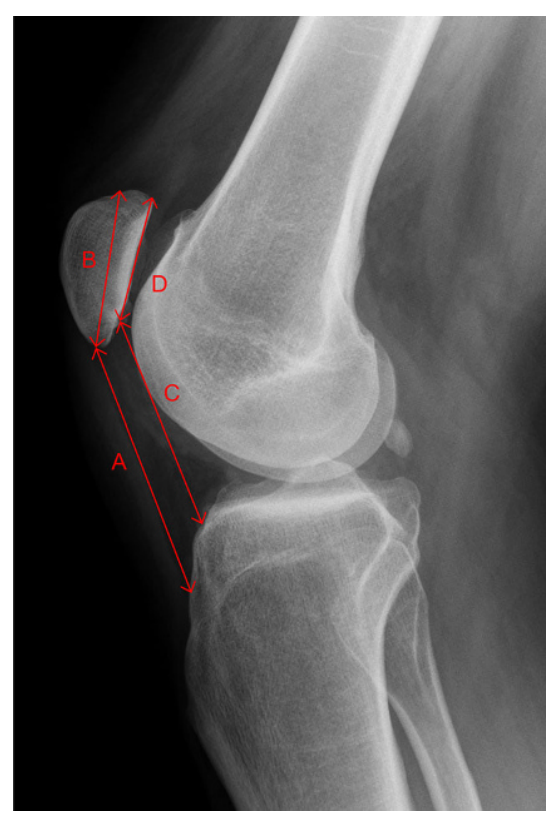

Abb. $1 \Delta$ Patella alta. Insall-Salvati-Ratio $($ ISR $)=A / B(A$ Länge des Lig. patellae, $B \mathrm{~Pa}$ tellalänge), Normwert: 0,6-1,2. Caton-Deschamps-Index $(\mathrm{CDI})=\mathrm{C} / \mathrm{D}(\mathrm{C}$ Strecke zwischen unterem Ende der Patellagelenkfläche und Tibiaplateau, $D$ Länge der Patellagelenkfläche), Normwert:0,6-1,2 

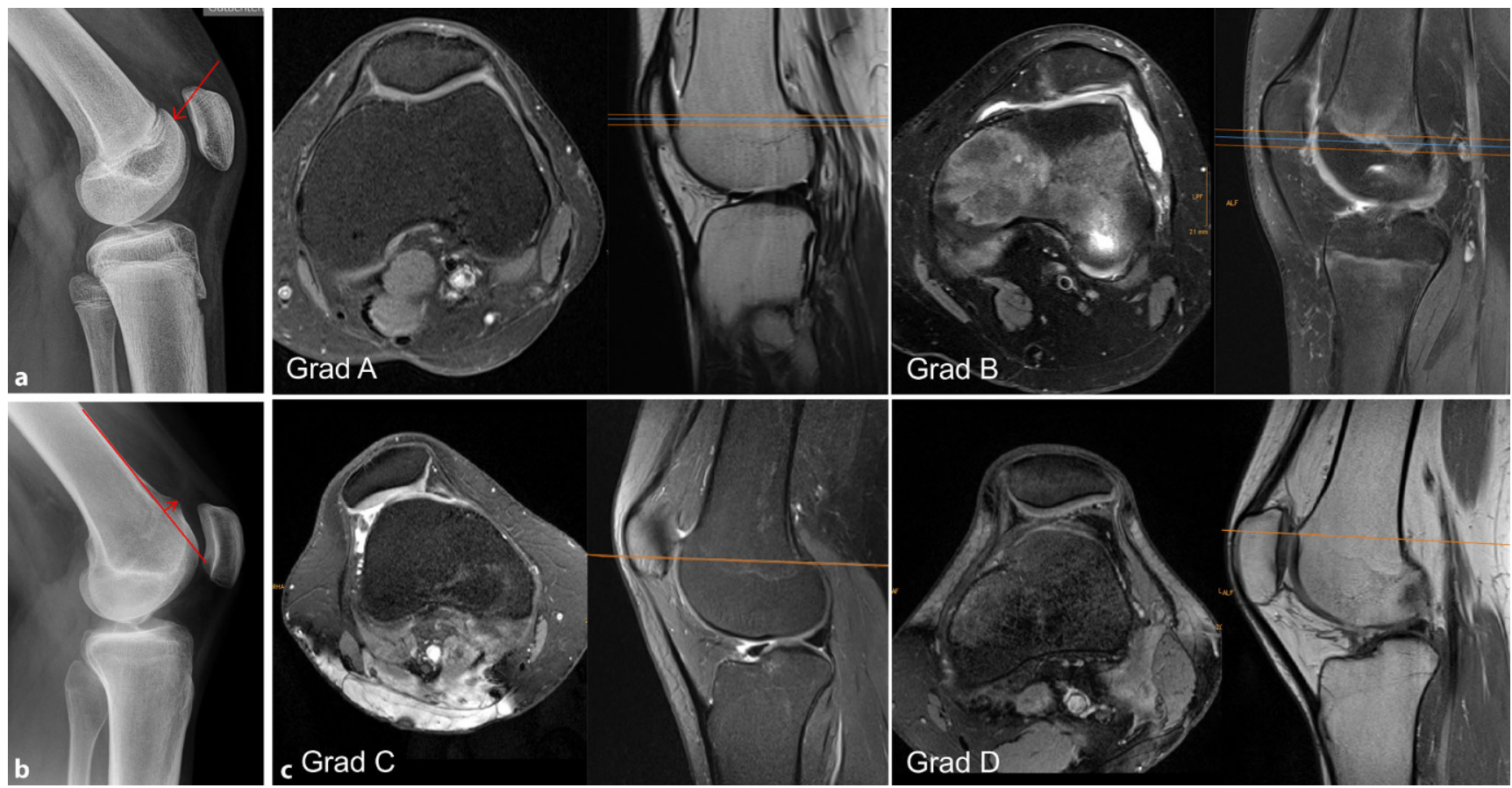

Abb. 2 ム Trochleadysplasie im seitlichen Röntgen und der Magnetresonanztomographie (MRT).a "Crossing sign“ als Hinweis auf eine Trocheladysplasie. Pfeil Überschneidung des Sulcus trochleae mit der lateralen Femurkondyle. $\mathbf{b}$ „Bump/trochlea offset": Erhebung der Trochlea über den anterioren Femurkortex (Normwert: $<5 \mathrm{~mm}$ ). c Trochleadysplasie in der MRT gemäß morphologischer Klassifikation nach Dejour in Grad A-D
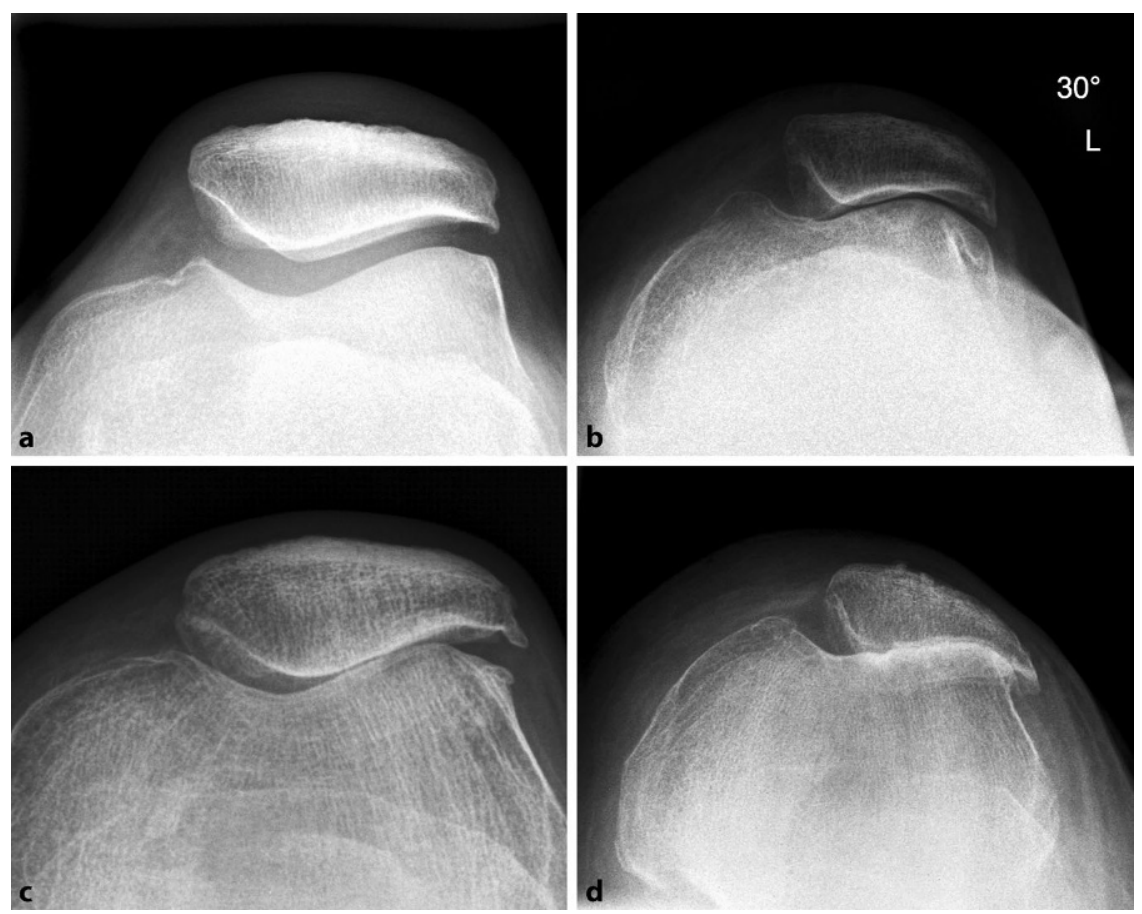

Abb. 3 A Iwano-Klassifikation der Arthrose des Patellofemoralgelenks (PFG). a Stadium 1: geringe Verschmälerung des Gelenkspalts ( $>3 \mathrm{~mm}$ an der engsten Stelle). b Stadium 2: moderate Verschmälerung des Gelenkspalts ( $<3 \mathrm{~mm}$ an der engsten Stelle). c Stadium 3: Knochen-Knochen-Kontakt auf $\leq 25 \%$ der Gelenkflächen. $\mathbf{d}$ Stadium 4: Knochen-Knochen-Kontakt auf > $25 \%$ der Gelenkflächen teren Ende der Patellagelenkfläche und dem Tibiaplateau (• Abb. 1). Allerdings variieren die in der Literatur angegebenen Grenzwerte für die Definition einer Patella alta erheblich (z. B. ISR: von $>1,2$ bis $>1,5$; CDI: von $>1,2$ bis $>1,3$; [3]). Während für die einzelnen Ratios eine sehr gute Intra- und Interobserverreliabilität gezeigt werden konnten, bestanden in zwei Drittel der Fälle durch Anwendung der verschiedenen Indices variable Ergebnisse in Bezug auf das Vorliegen einer Patella alta $[18,20]$.

Ein weiterer Risikofaktor für eine patellofemorale Instabilität, die Trochleadysplasie, kann in der seitlichen Aufnahme ebenfalls beurteilt werden. In der seitlichen Aufnahme wird auf eine Überschneidung des Trochleasulkus mit der lateralen Trochleaschulter (,crossing sign"; - Abb. 2a) sowie auf einen NiveauUnterschied der medialen und lateralen Trochleaschulter (Doppelkontur) geachtet. Zusätzlich wird die Erhöhung der Trochlea über den anterioren Kortex des Femurschafts („,bump“) gemessen (• Abb. 2b). 


\section{Patella tangential}

Die Tangentialaufnahme der Patella kann - wie in der seitlichen Aufnahme - Einsicht in den Gelenkspalt geben, sodass auch hier Frakturen und degenerativer Veränderungen detektiert werden können. Eine Arthrose des PFG wird in dieser Aufnahme üblicherweise nach der Iwano-Klassifikation eingeteilt (- Abb. 3; [9]). Auch Flake-Frakturen nach Patellaluxation kommen in der Tangentialaufnahme typischerweise gut zur Darstellung. Die Form der Patella kann in die Typen A bis C nach Wiberg eingeteilt werden [24]. Außerdem kann die Stellung der Patella in Bezug auf die Trochlea (patellofemorales Alignment bzw. Tracking) evaluiert werden. Eine Verschiebung der Kniescheibe in mediolateraler Richtung wird als PatellaShift bezeichnet. Zur Quantifizierung der Patellaverschiebung stehen neben älteren Messmethoden wie dem Kongruenzwinkel [13] heute verschiedene Messverfahren wie die axiale lineare Patellaverschiebung zu Verfügung [22]. Dabei wird der Abstand zwischen Patellafirst und Trochlearinne parallel zu einer Linie zwischen medialer und lateraler Trochleaschulter gemessen (• Abb. 4a). Die Kippung der Kniescheibe wird mit dem Patella-Tilt-Winkel beschrieben, dem Winkel zwischen Patella und Trochlealinie ( Abb.4b). Auch eine Trochleadysplasie kann in dieser Aufnahme entdeckt werden. Da für die deskriptive Klassifikation nach Dejour in früheren Studien nur eine geringe Intra- und Interobserverreliabilität demonstriert werden konnte [16], wurden zu deren Beurteilung auch objektive Parameter entwickelt. In der Literatur sind inzwischen 33 verschiedene Messmethoden beschrieben [15], u.a. der Sulkuswinkel. Er beschreibt den Winkel zwischen medialer und lateraler Trochleawand (- Abb.4c). Auch der lateralen TrochleaInklination [5], die durch eine Magnetresonanztomographie (MRT) bestimmt werden kann, wird eine wichtige Bedeutung zur objektivierbaren Beurteilung einer Trochleadysplasie zugeschrieben [15]. Kritisch anzumerken ist aber auch bei den objektiven Parametern, dass die Reliabilität insbesondere bei höhergradi-

Arthroskopie 2020 · 33:412-419 https://doi.org/10.1007/s00142-020-00385-z

(c) Der/die Autor(en) 2020

P. Kappel · M. Liebensteiner · AGA Komitee Knie-Patellofemoral

\section{Bildgebende Diagnostik patellofemoraler Störungen}

\section{Zusammenfassung}

Hintergrund. Patellofemorale Störungen sind häufig. Zur diagnostischen Abklärung gehört neben einer spezifischen Anamnese und detaillierten körperlichen Untersuchung auch die bildgebende Diagnostik. Das Ziel dieses Artikels ist es, die verschiedenen bildgebenden Techniken darzustellen, die zur Diagnostik patellofemoraler Störungen angewandt werden.

Material und Methoden. Es erfolgte eine Suche der in der Literatur beschriebenen bildgebenden Techniken sowie der darin ersichtlichen Befunde bei patellofemoralen Störungen.

Ergebnisse. Die Bildgebung des Patellofemoralgelenks muss sowohl eine Beurteilung von Traumafolgen bzw. einer Degeneration des Gelenks als auch eine Analyse der für die patellofemorale Instabilität relevanten anatomischen Risikofaktoren ermöglichen. Die Basisdiagnostik hierfür stellt das Röntgen in 3 Ebenen dar. Eine Ganzbeinstandaufnahme ermöglicht die Detektion von Achsfehlstellungen. Der Goldstandard zur
Bestimmung von Torsionsfehlern und dem TT-TG-Abstand (Distanz zwischen der Tuberositas tibiae und der Trochleagrube) ist die Computertomographie (CT). Die Magnetresonanztomographie (MRT) ermöglicht eine dreidimensionale Darstellung der Weichteilstrukturen wie dem medialen patellofemoralen Ligament (MPFL) sowie eine Beurteilung der Trochleamorphologie. Die SPECT-CT (,single photon emission computed tomography") zur Darstellung des Knochenstoffwechsels bleibt speziellen Fragestellungen vorbehalten.

Diskussion. Die bildgebende Diagnostik patellofemoraler Störungen ist multimodal. Entsprechend der Fragestellung reicht die Diagnostik von der alleinigen Basisdiagnostik bis hin zu innovativen Untersuchungstechniken wie der SPECT-CT.

\section{Schlüsselwörter}

Patellofemoralgelenk · Instabilität · Trauma . Röntgen · Magnetresonanztomographie

\section{Imaging diagnostics of patellofemoral disorders}

\section{Abstract}

Background. Patellofemoral disorders are frequent issues in orthopedics. Besides a specific medical history and detailed physical examination, the diagnostic clarification also involves imaging techniques. The aim of this article is to present the various imaging techniques used in the diagnostics of patellofemoral disorders.

Material and methods. A literature search was carried out for imaging techniques in relation to the findings in patellofemoral disorders.

Results. Imaging of the patellofemoral joint must enable assessment of trauma sequelae or degeneration of the joint as well analysis of the anatomic risk factors relevant for patellofemoral instability. The standard diagnostic procedure is radiographic imaging in three levels so that trauma sequelae, degenerative alterations and risk factors for patellofemoral instability can be assessed. Full length weight-bearing images enable the identification of axis malalignment. The gold standard to determine torsion errors and the distance between the tubercle of the tibia and the olecranon fossa (TT-TG) is computed tomography (CT). Magnetic resonance imaging (MRI) enables a threedimensional representation of soft tissue structures, such as the medial patellofemoral ligament (MPFL) as well as an assessment of trochlear morphology. Innovative techniques, such as single photon emission CT (SPECT-CT) to visualize bone metabolism are reserved for special questions.

Conclusion. The imaging diagnostics of patellofemoral disorders are multimodal. The diagnostics range from a standard radiographic basic diagnostics to innovative investigation techniques such as SPECT-CT depending on the problem in question.

\section{Keywords}

Patellofemoral joint · Instability · Trauma . $X$-ray $\cdot$ Magnetic resonance imaging 
ger Trochleadysplasie nur gering ist und eine klare Einordnung der Messwerte bislang noch nicht möglich ist [14]. Da die Patella-Tangentialaufnahme in $30^{\circ}$ oder $45^{\circ}$ Flexion durchgeführt wird, wird darüber hinaus nur die mittlere Trochlea abgebildet. Daher können Pathologien im Bereich der proximalen Trochlea übersehen werden, was den Stellenwert der seitlichen Röntgenaufnahme bzw. der Schnittbildgebung unterstreicht.

\section{Ganzbeinstandaufnahme}

In der Ganzbeinstandaufnahme kann eine verlässliche Evaluation der Beinachse stattfinden. Da es durch mangelhafte Einstellung zu großen Messabweichungen kommt, muss die Durchführung nach einem standardisierten Protokoll wie dem von Cooke et al. erfolgen [6]. Neben einer Messung der Beinachse können verschiedene Gelenkwinkel (medialer proximaler Tibiawinkel, MPTW; lateraler distaler Femurwinkel, LDFW) bestimmt werden, um eine Achsdeformität näher charakterisieren zu können ( $\bullet$ Abb. 5a). So kann beispielsweise eine Valgus-Beinachse als Ursache für eine patellofemorale Instabilität detektiert werden.

\section{Computertomographie}

Die Computertomographie (CT) hat einen großen Stellenwert in der Diagnostik des Patellofemoralgelenks. Neben der weiterführenden Diagnostik bei Frakturen wird sie als Standard für die Bestimmung des TT-TG-Abstands („tibial tubercle-to-trochlear groove“) eingesetzt und hat zur Feststellung möglicher Torsionsfehlstellungen des Oberund/oder Unterschenkels eine zentrale Bedeutung.

\section{Femorale Torsion}

Bei klinischem Verdacht auf Torsionsfehlstellungen (Kniescheibenstrabismus) sollte eine Torsionsdifferenz-CT durchgeführt werden. Die femorale Torsion ist die Verdrehung zwischen dem proximalen und distalen Anteil des Femurs in der transversalen Ebene. Sie ist definiert als der Winkel zwischen posteriorer Kondylenlinie des Femurs und Schenkelhals
Hier steht eine Anzeige. Springer 

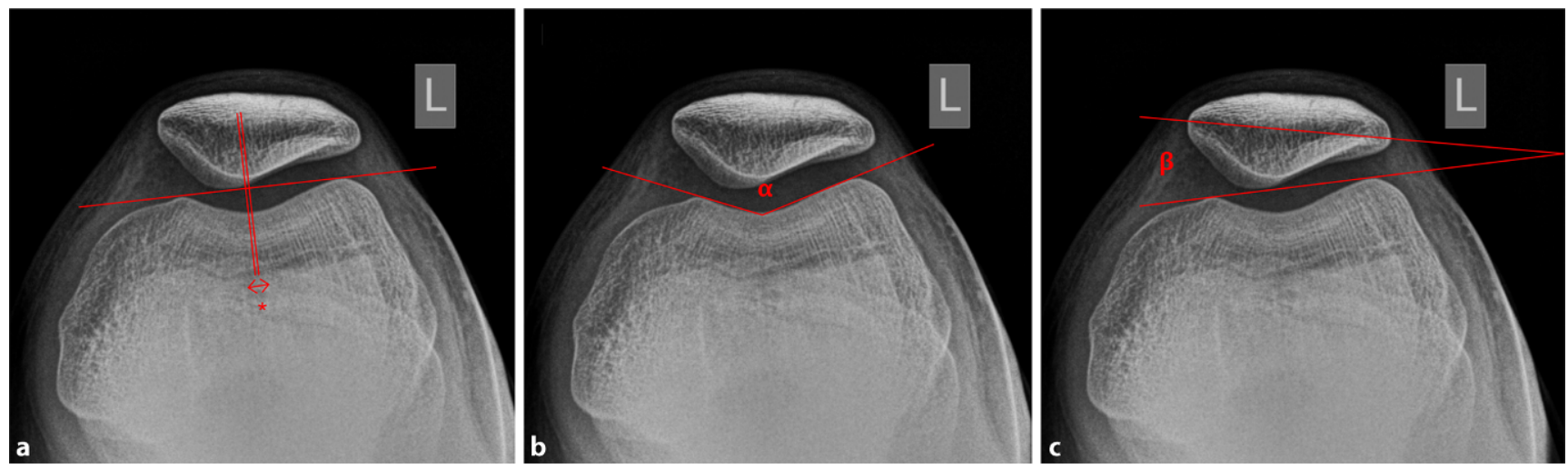

Abb. $4 \Delta$ a Bestimmung des Patella-Shift mithilfe der linearen Patellaverschiebung: Abstand zwischen Patellafirst und Sulcus trochleae, gemessen parallel zur Linie zwischen medialer und lateraler Trochleaschulter. (Stern, Normwert: $\leq 2 \mathrm{~mm}$ ). b Sulkuswinkel zur Beurteilung einer Trochleadysplasie: Winkel zwischen medialer und lateraler Trochleawand ( $a$, Normwert: $<145^{\circ}$ ). c Patella-Tilt-Winkel: Winkel zwischen Patella und Trochlealinie $\left(\beta\right.$, Normwert: $\left.\leq 5^{\circ}\right)$

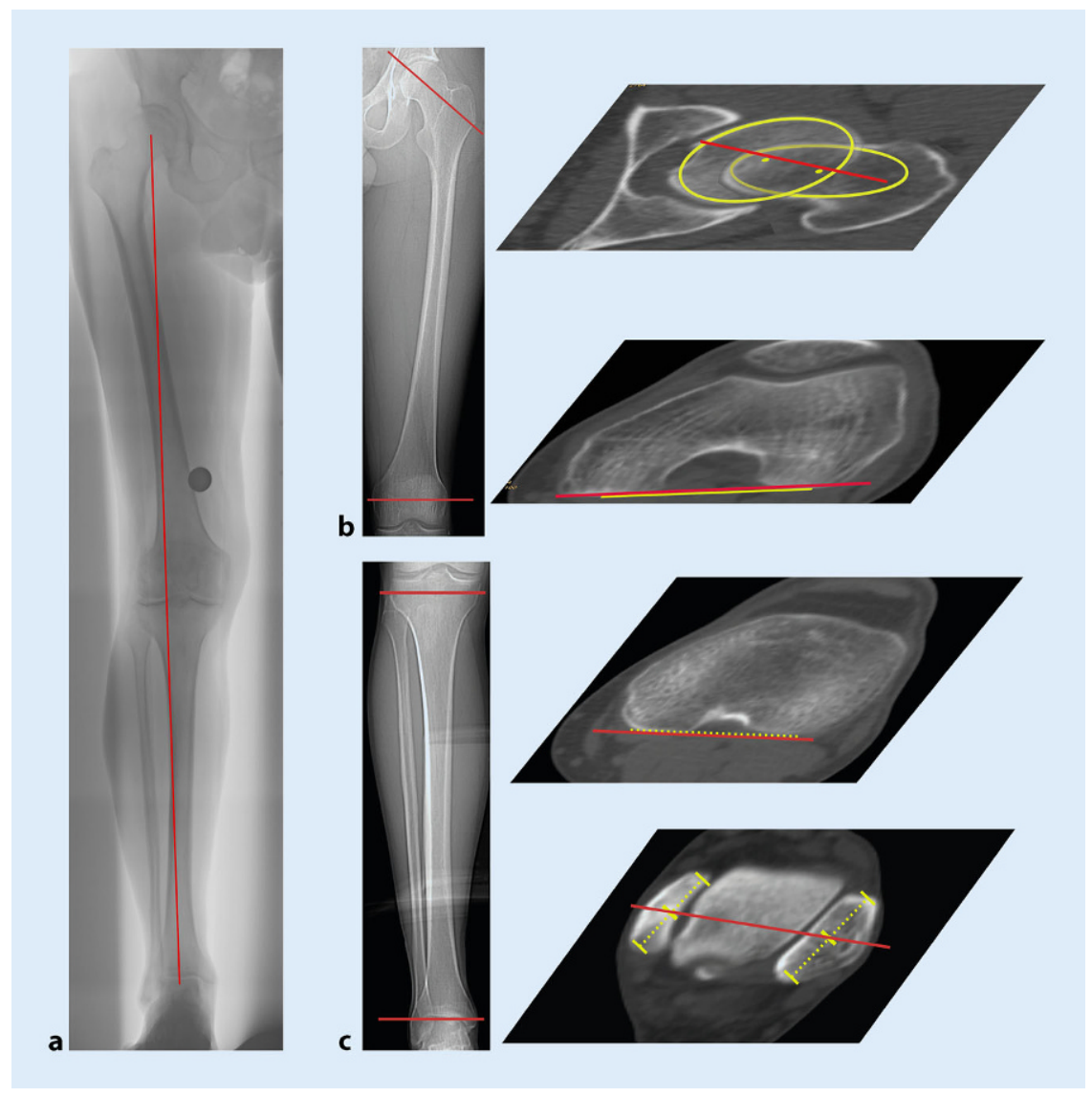

Abb. $5 \Delta$ a Bestimmung der mechanischen Beinachse (Verbindungslinie zwischen Hüftkopfzentrum und Zentrum der Sprunggelenksgabel). In diesem Beispiel läuft die Belastungsachse lateral durch das Kniegelenk, entsprechend einer Valgusdeformität. b Femurtorsion (Winkel zwischen Femurhals und dorsaler Kondylenlinie des Femurs). c Tibiatorsion (Winkel zwischen dorsaler Kondylenlinie der Tibia und Sprunggelenkachse)
(- Abb. 5b). Zur Beurteilung wurden verschiedene Messmethoden publiziert, die alle eine hohe Reliabilität aufweisen. Eine Kenntnis der verschiedenen Messmethoden ist aber essenziell, da sie auch mit unterschiedlichen Normwerten einhergehen [11]. Während Waidelich et al. einen Normwert von $22,4^{\circ} \pm 6,8^{\circ}$ definierten, liegt dieser bei der Messmethode nach Hernandez et al. bei $11,4^{\circ} \pm 7,4^{\circ}$ [11]. Werte unter $0^{\circ}$ und über $30^{\circ}$ Innentorsion gelten bei Erwachsenen in allen Publikationen als pathologisch.

\section{Tibiale Torsion}

Die Tibiatorsion ist die Verdrehung zwischen dem proximalen und distalen Anteil der Tibia in der transversalen Ebene. Jakob et al. [10] beschrieben 1980 die erste CT-basierte Messmethode. Seitdem wurden multiple Messverfahren publiziert. In der Mehrzahl der Fälle wird der Winkel zwischen posteriorer Kondylenlinie der Tibia und Sprunggelenkachse (Linie zwischen Innen- und Außenknöchel) zugrunde gelegt (• Abb. 5c). Wie bei der Femurtorsion findet sich in der Literatur eine große physiologische Streubreite, wobei Werte unter $10^{\circ}$ und über $40^{\circ}$ Außentorsion übereinstimmend als pathologisch angesehen werden [23].

\section{TT-TG-Abstand}

Der TT-TG-Abstand ist die Distanz zwischen der Tuberositas tibiae und dem Sulcus trochleae in Millimetern, wobei die 

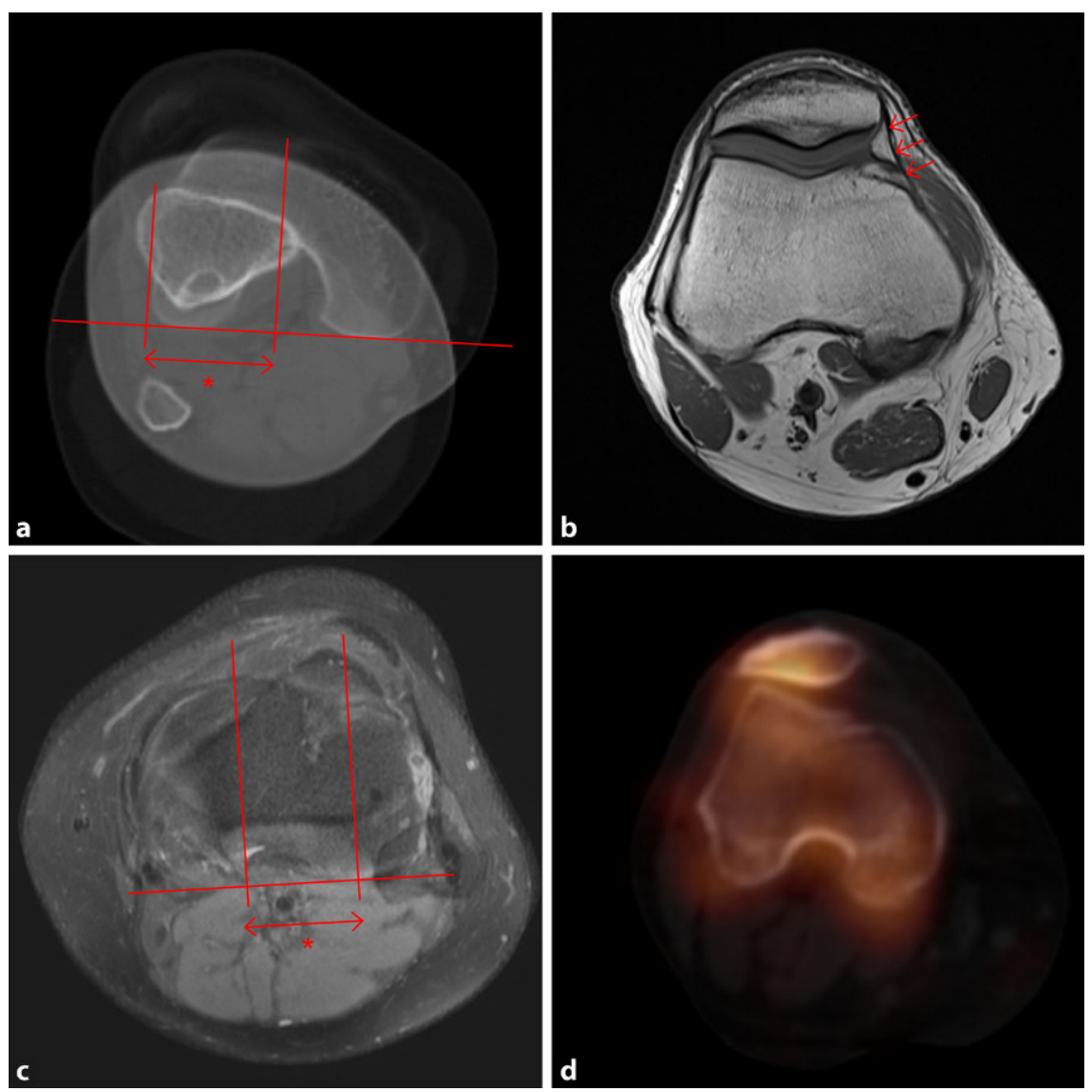

Abb. $6 \Delta$ a Messung des TT-TG-Abstands („tibial tubercle-to-trochlear groove“) in der Computertomographie (Stern; Normwert: $<20 \mathrm{~mm}$ ). b Messung des TT-PCL-Abstandss in der Magnetresonanztomographie (Normwert: $<24 \mathrm{~mm}$ ). c Mediales patellofemorales Ligament (MPFL) in der MRT (rote Pfeile). d SPECT-CT (,single photon emission computed tomography “): Mehranreicherung im Bereich der lateralen Patellafacette bei lateraler, patellofemoraler Hyperkompression

Messung parallel zur posterioren Kondylenlinie des Femurs erfolgt (• Abb.6a). Für diesen Parameter sind in der Literatur unterschiedliche Grenzwerte beschrieben, wobei die Definition des pathologischen Grenzwerts zwischen $>14$ und $>20 \mathrm{~mm}$ variiert.

\section{》) Goldstandard zur Messung des TT-TG-Abstands ist die Computertomographie}

Zur Bestimmung des TT-TG-Abstands werden entweder CT oder MRT herangezogen, wobei die CT den Goldstandard darstellt. Hier ist allerdings zu beachten, dass es aufgrund lagerungsbedingt variierender Beugegrade in der CT bzw. MRT zu Messdifferenzen des TT-TGAbstands kommen kann. $\mathrm{Zu}$ den Messwerten der TT-TG-Distanz aus der MRT sollten $3 \mathrm{~mm}$ addiert werden, um sie mit üblichen CT-Werten vergleichen zu können [19].

\section{SPECT-CT}

Für spezielle Fragestellungen in Hinblick auf das PFG kann die ,single photon emission computed tomography" (SPECT-CT) diagnostisch hilfreich sein [8]. Der Vorteil dieser Methode liegt in der Fusion aus Informationen zum Knochenstoffwechsel (Tc-RadiotracerUptake) und herkömmlicher CT. Das dreidimensionale Bild ermöglicht im Gegensatz zur frontalen Aufsicht im Szintigramm eine exzellente Beurteilung des PFG. Bei Verdacht auf eine pathologische Biomechanik im PFG kann dieser durch eine Stoffwechselsteigerung an entsprechender Lokalisation erhärtet werden (z.B. gesteigerte Stoffwechselaktivität bei lateraler, patellofemoraler Hyperkompression; - Abb. 6d). Aller- dings sollte bedacht werden, dass diese Untersuchungstechnik mit einer Strahlenbelastung einhergeht, welche einer CT des Thorax entspricht. Daher sollte sie bei jüngeren Patienten mit Zurückhaltung eingesetzt werden.

\section{Magnetresonanztomographie}

Die MRT stellt aufgrund der guten Weichteildarstellung, einhergehend mit einer dreidimensionalen Darstellung des Gelenks eine ausgezeichnete Modalität zur Beurteilung des PFG dar und ist inzwischen unverzichtbar geworden. Die Standarduntersuchung sollte immer eine Sequenz mit Fettsuppression beinhalten, um Ergüsse oder Knochenmarködeme abbilden zu können [21].

\section{Mediales patellofemorales Ligament}

Das mediale patellofemorale Ligament (MPFL), der wichtigste passive Stabilisator der Kniescheibe, lässt sich in der MRT als hypointense Struktur von seinem Ursprung am medialen Patellarand bis zum Ansatz am medialen Femurkondylus darstellen ( $\bullet$ Abb. 6b). Bei einer MPFL-Ruptur nach Patellaluxation kann in der MRT auch eine Aussage über den Ort der Ruptur getroffen und operative Maßnahmen entsprechend geplant werden. An dieser Stelle soll festgehalten werden, dass nicht immer nur die radiologisch verifizierte MPFL-Ruptur (Diskontinuität von Fasern) die Indikation zur MPFL-Rekonstruktion begründet. Vielmehr kann auch eine chronische MPFL-Insuffizienz (z. B. bei koexistierender Trochleadysplasie) die Indikation zur MPFL-Rekonstruktion bedeuten.

\section{Quadrizepssehne}

Die Quadrizepssehne setzt als gemeinsameSehne der 4 Muskelanteile des M. quadriceps femoris am oberen Pol der Patella an. Während der oberflächliche Anteil extraartikulär liegt, befindet sich der tiefe Sehnenanteil zumindest teilweise intraartikulär. Bei physiologischer Biomechanik ist der M. quadriceps femoris der stärkste aktive Stabilisator der Patella, er 
kann bei ausgeprägter femoraler Antetorsion aber auch eine Luxation begünstigen. In der MRT können Verletzungen oder Überlastungen der einzelnen Muskeln oder der Sehne selbst exzellent nachgewiesen werden.

\section{Patellasehne}

Die Patellasehne verbindet die Patella mit der Tibia. Sie ist im Wesentlichen eine Fortsetzung von Sehnenfasern des M. rectus femoris, entspringt am unteren Pol der Patella und reicht bis zur Tuberositas tibiae. Während eine komplette Ruptur klinisch leicht zu diagnostizieren ist, besteht der Vorteil der MRT im Nachweis von Partialrupturen und Mikrotraumen. Hierbei kommt es zum Signalanstieg der normalerweise als hypointens imponierenden Patellasehne.

\section{Knorpel}

Während die Diagnostik der Arthrose des PFG eine Domäne der Nativradiologie ist, erlaubt das MRT auch eine Beurteilung geringerer Knorpelschäden. Der hyaline Knorpel kann hier sowohl bezüglich seiner Dicke als auch Oberflächenbeschaffenheit evaluiert werden. Außerdem lässt sich der subchondrale Knochen beurteilen. Zur Einteilung von Knorpelschäden findet zumeist die Klassifikation der ICRS (International Cartilage Repair Society) Anwendung:

- Grad I: oberflächliche Läsionen des Knorpels

- Grad II: Läsionen mit einer maximalen Tiefenausdehnung von $<50 \%$ der Knorpeldicke

- Grad III: Läsionen mit einer maximalen Tiefenausdehnung $>50 \%$ der Knorpeldicke

- Grad IV: osteochondrale Läsionen

Neben degenerativen Knorpelschäden lassen sich auch traumatische Knorpelschäden bzw. Knorpel-Knochen-Flakes, z.B. nach Patellaluxation, exzellent darstellen.

\section{Knochen}

Zum Nachweis von Frakturen stellt das Nativröntgen bzw. die CT nach wie vor den Goldstandard dar. Mittels MRT können jedoch auch Mikrofrakturen und Knochenkontusionen nachgewiesen werden. Hier zeigt die MRT eine höhere Sensitivität als die anderen bildgebenden Verfahren. Frakturen von Trabekeln und knöcherne Einblutungen stellen sich als Knochenmarködem dar, welches zwar unspezifisch ist, zusammen mit der Anamnese allerdings meist eindeutig als Kontusion („bone bruise“) identifiziert werden kann. Nach Patellaluxation zeigt sich typischerweise ein Ödem im Bereich der lateralen Femurkondyle und am medialen Patellapol. Bei atraumatischen Beschwerden kann ein solches Ödem auch überlastungsassoziiert sein (Stressödem). Beispiele hierfür sind ein Ödem im medialen Kompartiment bei Varusfehlstellung oder der Patella beim Patellaspitzensyndrom.

\section{TT-PCL-Abstand}

Zur Identifikation einer pathologisch lateralisierten Tuberositas tibiae kann der TT-TG-Abstand auch in der MRT gemessen werden. Hier ist allerdings $\mathrm{zu}$ beachten, dass die MRT im Vergleich zur CT meistens in Beugestellung durchgeführt wird und der TT-TG-Abstand deshalb eher unterschätzt wird [1]. In der MRT steht uns mit dem TT-PCL-Abstand („tibial tubercle-to-posterior cruciate ligament") außerdem ein weiterer Parameter zur Beurteilung einer Lateralisation der Tuberositas tibiae zur Verfügung. Er ist definiert als der Abstand zwischen der medialen Begrenzung des hinteren Kreuzbands und der Tuberositas tibiae (• Abb. 6c; [19]). Dieser Parameter scheint v.a. dann überlegen zu sein, wenn die zweifelsfreie Identifikation der Trochlearinne aufgrund einer Trochleadysplasie erschwert ist. Außerdem ist der Parameter unabhängig von der Rotation des Kniegelenks und insbesondere in der häufig ohnehin vorliegenden MRT sicher zu bestimmen. Ein TT-PCL-Abstand $\geq 24 \mathrm{~mm}$ gilt als pathologisch [19].

\section{Patellahöhe}

Messungen der Patellahöhe können auch in der MRT mithilfe der oben beschriebenen Indices vorgenommen werden. $\mathrm{Zu}$ beachten ist allerdings, dass MRT-basierte Messungen höhere Werte als im Röntgen ergeben mit durchschnittlichen Differenzen von 0,18 (CDI) bzw. 0,11 (ISR). In der MRT steht uns mit dem PatellaTrochlea-Index nach Biedert eine weitere Methode zur Bestimmung der Patellahöhe zur Verfügung [2]. Vorteil dieses Verfahrens ist, dass die Patellahöhe in Bezug auf die Trochlea (den eigentlichen Gelenkpartner) untersucht wird und nicht - wie bei den übrigen Indices - auf die Tibia bezogen wird.

\section{Trochleadysplasie}

Die MRT ist zusammen mit dem Röntgen im seitlichen Strahlengang der Goldstandard zur Bestimmung der Trochleamorphologie. Zur Einteilung wird überwiegend die Klassifikation nach Dejour herangezogen, welche die verschiedenen morphologischen Varianten der dysplastischen Trochlea beschreibt (- Abb. 2c; [7]).

\section{Torsion}

Die Messung der Torsion der unteren Extremität ist eine Domäne der CT. Aber auch in der MRT können Torsionsdifferenzen ermittelt werden [17]. Dies ist v. a. bei dem meist jungen Patientenkollektiv mit patellofemoraler Instabilität zu bedenken. Allerdings dürfen die hier erhobenen Messwerte nicht mit CT-basierten Messungen verglichen werden. So zeigten Botser et al.zwar eine hohe Reliabilität für Messungen der femoralen Torsion in der MRT, allerdings waren die Messwerte im Vergleich zur CT durchschnittlich $8,9^{\circ}$ geringer [4].

\section{Fazit für die Praxis}

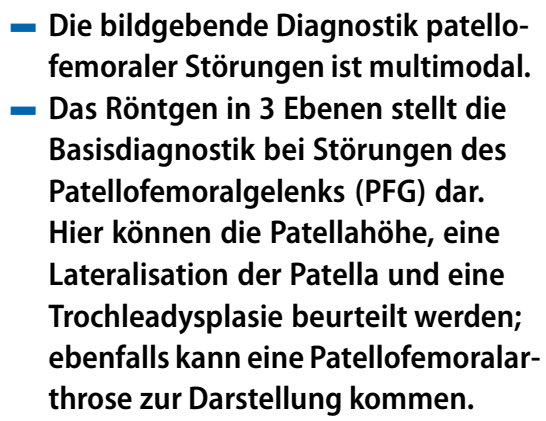




\section{- In einer Ganzbeinstandaufnahme können sowohl die Beinachse als auch verschiedene Gelenkwinkel (LDFW, MPTW) ausgemessen werden. \\ - Die MRT dient der Darstellung der Weichteile. \\ - In der MRT ist eine Beurteilung von MPFL, Trochlea, Knorpel oder einem Knochenmarködem sowie eine Messung des TT-PCL-Abstands möglich. \\ - Als Alternative zur CT können hier auch der TT-TG-Abstand und die Torsion von Ober- und Unterschenkel bestimmt werden. \\ - Die CT ist der Goldstandard zur Mes- sung des TT-TG-Abstands und von femoralen und tibialen Torsionsfehl- stellungen. \\ - Spezielle Untersuchungstechniken wie die SPECT-CT sind besonderen Fragestellungen vorbehalten.}

\section{Korrespondenzadresse}

\section{Liebensteiner}

Universitätsklinik für Orthopädie Innsbruck, Medizinische Universität Innsbruck Anichstr. 35, 6020 Innsbruck, Österreich michael.liebensteiner@i-med.ac.at

Funding. Open access funding provided by University of Innsbruck and Medical University of Innsbruck.

\section{Einhaltung ethischer Richtlinien}

Interessenkonflikt. P. Kappel und M. Liebensteiner geben an, dass kein Interessenkonflikt besteht.

Für diesen Beitrag wurden von den Autoren keine Studien an Menschen oder Tieren durchgeführt. Für die aufgeführten Studien gelten die jeweils dort angegebenen ethischen Richtlinien.

Open Access. Dieser Artikel wird unter der Creative Commons Namensnennung 4.0 International Lizenz veröffentlicht, welche die Nutzung, Vervielfältigung, Bearbeitung, Verbreitung und Wiedergabe in jeglichem Medium und Format erlaubt, sofern Sie den/die ursprünglichen Autor(en) und die Quelle ordnungsgemäß nennen, einen Link zur Creative Commons Lizenz beifügen und angeben, ob Änderungen vorgenommen wurden.

Die in diesem Artikel enthaltenen Bilder und sonstiges Drittmaterial unterliegen ebenfalls der genannten Creative Commons Lizenz, sofern sich aus der Abbildungslegende nichts anderes ergibt. Sofern das betreffende Material nicht unter der genannten Creative Commons Lizenz steht und die betreffende Handlung nicht nach gesetzlichen Vorschriften erlaubt ist, ist für die oben aufgeführten Weiterverwendungen des Materials die Einwilligung des jeweiligen Rechteinhabers einzuholen.

Weitere Details zur Lizenz entnehmen Sie bitte der Lizenzinformation auf http://creativecommons.org/ licenses/by/4.0/deed.de.

\section{Literatur}

1. Becher C, Fleischer B, Rase M et al (2017) Effects of upright weight bearing and the knee flexion angle on patellofemoral indices using magnetic resonance imaging in patients with patellofemoral instability. Knee Surg Sports Traumatol Arthrosc. https://doi.org/10.1007/s00167-015-3829-8

2. Biedert RM, Albrecht S (2006) The patellotrochlear index: a new index for assessing patellar height. Knee Surg Sports Traumatol Arthrosc. https://doi. org/10.1007/s00167-005-0015-4

3. Biedert RM, Tscholl PM (2017) Patella Alta: a comprehensive review of current knowledge. Am J Orthop 46(6):290-300

4. Botser IB, Ozoude GC, Martin DE et al (2012) Femoral anteversion in the hip: Comparison of measurement by computed tomography, magnetic resonance imaging, and physical examination. J Arthrosc Relat Surg. https://doi. org/10.1016/j.arthro.2011.10.021

5. Carrillon Y, Abidi H, Dejour D et al (2000) Patellar instability: assessment on MR images by measuring the lateral trochlear inclination - initial experience. Radiology. https://doi.org/10.1148/ radiology.216.2.r00au07582

6. Cooke TDV, Sled EA, Scudamore RA (2007) Fronta plane knee alignment: a call for standardized measurement. JRheumatol 34:1796-1801

7. Dejour DLCB (2007) Osteotomies in patello-femoral instabilities. Sports Med Arthrosc 15(1):39-46

8. Hirschmann MT, Amsler F, Rasch H (2015) Clinical value of SPECT/CT in the painful total knee arthroplasty (TKA): a prospective study in a consecutive series of 100 TKA. Eur J Nucl Med Mol Imaging. https://doi.org/10.1007/s00259-0153095-5

9. Iwano $\mathrm{T}$, Kurosawa $\mathrm{H}$, Tokuyama $\mathrm{H}$, Hoshikawa $\mathrm{Y}$ (1990) Roentgenographic and clinical findings of patellofemoral osteoarthrosis. With special reference to its relationship to femorotibial osteoarthrosis and etiologic factors. Clin Orthop Relat Res. https://doi.org/10.1097/00003086199003000-00028

10. Jakob RP, Haertel M, Stussi E (1980) Tibial torsion calculated by computerised tomography and compared to other methods of measurement. J Bone Joint Surg Br. https://doi.org/10.1302/ 0301-620x.62b2.7364840

11. KaiserP,AttalR, KammererMetal (2016)Significant differences in femoral torsion values depending on the CT measurement technique. Arch Orthop Trauma Surg. https://doi.org/10.1007/s00402016-2536-3

12. Koëter S, Bongers EMHF, De Rooij J, Van Kampen A (2006) Minimal rotation aberrations cause radiographic misdiagnosis of trochlear dysplasia. Knee Surg Sports Traumatol Arthrosc. https://doi.org/ 10.1007/s00167-005-0031-4

13. Merchant AC, Mercer RL, Jacobsen $\mathrm{RH}$, Cool CR (1974) Roentgenographic analysis of patellofemoral congruence. J Bone Joint Surg Am. https://doi. org/10.2106/00004623-197456070-00007
14. Nelitz M, Lippacher S, Reichel H, Dornacher D (2014) Evaluation of trochlear dysplasia using MRI: Correlation between the classification system of Dejour and objective parameters of trochlear dysplasia. Knee Surg Sports Traumatol Arthrosc. https://doi.org/10.1007/s00167-012-2321-y

15. Paiva M, Blønd L, Hölmich P et al (2018) Quality assessment of radiological measurements of trochlear dysplasia; a literature review. Knee Surg Sports Traumatol Arthrosc. https://doi.org/10. 1007/s00167-017-4520-z

16. Rémy F, Chantelot $C$, Fontaine $C$ et al (1998) Interand intraobserver reproducibility in radiographic diagnosis and classification of femoral trochlear dysplasia. Surg Radiol Anat. https://doi.org/10. 1007/BF01628492

17. Schneider B, Laubenberger J, Jemlich $S$ et al (1997) Measurement of femoral antetorsion and tibial torsion by magnetic resonance imaging. Br J Radiol. https://doi.org/10.1259/bjr.70.834. 9227249

18. Seil R, Müller B, Georg T et al (2000) Reliability and interobserver variability in radiological patellar heightratios. KneeSurg Sports Traumatol Arthrosc https://doi.org/10.1007/s001670000121

19. Seitlinger G, Scheurecker G, Högler R et al (2012) Tibial tubercle-posterior cruciate ligament distance: a new measurement to define the position of the tibial tubercle in patients with patellar dislocation. Am J Sports Med. https://doi. org/10.1177/0363546512438762

20. Smith TO, Davies L, Toms AP et al (2011) The reliability and validity of radiological assessment for patellar instability. A systematic review and meta-analysis. Skeletal Radiol. https://doi.org/10. 1007/s00256-010-0961-x

21. Thomas S, Rupiper D, Stacy GS (2014) Imaging of the patellofemoral joint. Clin Sports Med 33:413-436. https://doi.org/10.1016/j.csm.2014. 03.007

22. Urch SE, Tritle BA, Shelbourne DK, Gray T (2009) Axial linear patellar displacement: a new measurement of patellofemoral congruence. Am J Sports Med. https://doi.org/10.1177/ 0363546508328596

23. Waldt S, Eiber M, Wörtler M (2011) Messverfahren und Klassifikationen in der muskuloskelettalen Radiologie. Thieme, Stuttgart. https://doi.org/10. 1055/b-004-140285

24. Wiberg G (1941) Roentgenographic and anatomic studies on the patellofemoral joint. Acta Orthop Scand 12:319-409. https://doi.org/10.3109/ 17453674108988818 